\section{Electronic Medical Records: Unintended Consequences of a Duplicitous Default Setting}

To the Editor-Septicemia is a potentially life-threatening disorder, and physicians have a low threshold for obtaining blood samples for culture from patients with suspected septicemia. The standard of care is to draw 2 sets of blood samples for culture, both to increase the yield of true pathogens and to assist in determining whether a single positive result is caused by a contaminant rather than a true infection. One "set" is defined as the volume of blood obtained in a single venipuncture and inoculated into an aerobic and an anaerobic culture bottle.

After the introduction of an electronic medical record (EMR) system at our institution in March 2009, we noticed that the number of single-set blood cultures increased. In January 2009, before the introduction of the EMR system, $36(2.8 \%)$ of 1,277 blood cultures in our institution were single-set blood cultures. In July 2009, 106 (9.4\%) of 1,132 blood cultures were single-set blood cultures, a more than $300 \%$ increase that would indicate approximately 1,200 single-set blood cultures annually. On the basis of our observation and preliminary findings, we investigated the factors associated with the increase in ordering of single sets of blood samples for culture. We found that the EMR system was set with a default to order a single set of blood samples for culture, combined with a built-in warning of duplicate ordering when the physician attempted to order a second set. This combination discouraged the practitioner from ordering 2 sets of blood culture samples from the same patient during a 24 -hour period. Once we identified the issue with the duplicate warning in the EMR system, we requested that Information Technology Department staff change the blood culture order default setting to 2 sets of blood culture samples and eliminate the duplicate warning. The change was implemented at the end of October 2009. After the change, in November 2009, 46 (3.7\%) of 1,231 blood cultures ordered were single-set blood cultures, and in December 2009, 36 (3.1\%) of 1,171 blood cultures ordered were single-set blood cultures.

The EMR system default to order a single set of blood culture samples, combined with a warning of duplicate ordering, created 2 problems: a decrease in the yield of true positive results due to a decreased blood volume cultured and an inability to determine whether a positive blood culture result was caused by a contaminant. Numerous studies have documented the increased yield with increasing blood volume cultured. In 1983, Weinstein et al $^{1}$ reported results from 282 patients using 15-mL blood specimens. The cumulative yield of pathogens was $91 \%$ from the first culture and more than $99 \%$ from 2 cultures performed using separate specimens. In a study by Weinstein et al, ${ }^{1}$ blood cultures were performed using a manual blood culture system. In 2004, Cockrill et al ${ }^{2}$ reported a study involving 163 patients for whom blood cultures were performed using $20 \mathrm{~mL}$ of volume in a continuousmonitoring blood culture system. In their study, the yield of pathogens was $65 \%$ from the first culture, $80 \%$ from 2 cultures using separate specimens, and $96 \%$ from 3 cultures using separate specimens.

The number of blood culture sets that grow a particular microorganism, especially when measured as a function of the total number of blood culture sets obtained, has proved to be a useful aid in interpreting the clinical importance of positive blood culture results. ${ }^{1,3,4}$ Positive blood culture results caused by contamination with skin flora at the time of sample acquisition are common, representing up to one-half of all positive blood culture results at some healthcare facilities. ${ }^{1,3}$ Contaminants may in turn lead to unnecessary antibiotic therapy, additional testing and consultation, and increased length of stay. In true bloodstream infections, either all or most of the blood culture samples obtained will yield positive results; when a blood culture sample is contaminated, usually only 1 of several blood culture sets will yield positive results. However, this diagnostic maxim has no utility if only a single blood culture sample is obtained. In 1998, Schiffman et $\mathrm{al}^{5}$ reported a median adult inpatient blood culture contamination rate of $2.5 \%$. Using this estimate, the probability of recovering the same microorganism in 2 culture sets from 1 patient and of that organism being a contaminant is less than 1 in $1,000(0.025 \times 0.025=0.000625)$. The clinician can be quite confident, then, that a finding of 2 of 2 blood cultures positive for the same pathogen, even a pathogen that is commonly a contaminant, represents real disease, assuming that the 2 blood culture samples were obtained from separate venipunctures. If only a single blood culture sample is obtained, the value of this tool ceases to exist; and this is but one reason (another being increased blood volume) that the use of at least 2 blood culture specimens is recommended as standard practice. ${ }^{6,7,8}$

The EMR system is undoubtedly a useful new tool in our ever-advancing medical technology. The EMR system has the potential to improve efficiency, reduce cost, improve patient safety, and promote evidence-based medicine. Our case highlights the fact that defaults can be set to inadvertently direct physicians to provide substandard care. Physician users play an important role in the recognition and correction of design faults within an EMR system. Although constant efforts to improve the EMR system are being made by our information technology staff, it is still not a perfect system. We urge phy- 
sicians using any EMR system to be vigilant and provide constant feedback to improve this vital tool.

\section{ACKNOWLEDGMENTS}

Potential conflicts of interest. Both authors report no conflicts of interest relevant to this article.

Tariq Iqbal, MD; Edward C. Oldfield III, MD

From the Division of Infectious Diseases, Department of Medicine, Eastern Virginia Medical School, Norfolk, Virginia (both authors).

Address reprint requests to Tariq Iqbal, MD, Eastern Virginia Medical School, 825 Fairfax Avenue, Suite 410, Norfolk, VA 23507 (bluestar123@ gmail.com).

Infect Control Hosp Epidemiol 2010; 31(11):1198-1199

(C) 2010 by The Society for Healthcare Epidemiology of America. All rights reserved. 0899-823X/2010/3111-0018\$15.00. DOI: $10.1086 / 657073$

\section{REFERENCES}

1. Weinstein MP, Reller LB, Murphy JR, Lichtenstein KA. The clinical significance of positive blood cultures: a comprehensive analysis of $500 \mathrm{ep}$ isodes of bacteremia and fungemia in adults. I. Laboratory and epidemiologic observations. Rev Infect Dis 1983;5:35-53.

2. Cockerill FR III, Wilson JW, Vetter EA, et al. Optimal testing parameters for blood cultures. Clin Infect Dis 2004;38:1724-1730.

3. Weinstein MP, Towns ML, Quartey SM, et al. The clinical significance of positive blood cultures in the 1990s: a prospective comprehensive evaluation of the microbiology, epidemiology, and outcome of bacteremia and fungemia in adults. Clin Infect Dis 1997;24:584-602.

4. MacGregor RR, Beaty HN. Evaluation of positive blood cultures: guidelines for early differentiation of contaminated from valid positive cultures. Arch Intern Med 1972;130:84-87.

5. Schifman RB, Strand CL, Meier FA, Howanitz PJ. Arch Pathol Lab Med 1998;122(3):216-221.

6. Aronson MD, Bor DH. Blood cultures. Ann Intern Med 1987;106:246253.

7. Reimer LG, Weinstein MP, Wilson ML. Update on the detection of bacteremia and fungemia. Clin Microbiol Rev 1997;10:444-465.

8. Weinstein MP. Current blood culture methods and systems: clinical concepts, technology, and interpretation of results. Clin Infect Dis 1996;23: $40-46$.

\section{Extrinsic Contamination of Liquid Soap with Various Gram-Negative Bacteria in a Hospital in Turkey}

To the Editor-Because washing hands before and after direct contact with patients is the major component of infection control programs, microbial contamination of hand-washing soaps used in the hospital setting can present a challenge for infection control. Potentially pathogenic microorganisms, including diphtheroids, staphylococci, Escherichia coli, and Klebsiella, Pseudomonas, Serratia, Aspergillus, and Candida species, were found in bar soaps and their containers. ${ }^{1,2}$ Not only bar soaps but also liquid soaps can be contaminated intrinsically during the manufacture or extrinsically during use, particularly by gram-negative bacteria. ${ }^{3,4}$ Staphylococci are isolated more often from bar soaps rather than liquid soaps. Gram-negative bacteria are isolated from liquid soaps, including those that contain antibacterials. ${ }^{3-5}$ Klebsiella pneumoniae contaminated chlorhexidine-containing soap, Pseudomonas aeruginosa contaminated triclosan-containing soap, and Serratia marcescens contaminated chlorxylenol-containing soap, in several studies investigating infection outbreaks. ${ }^{3,4,6} \mathrm{~S}$. marcescens was associated with hospital infections and infection outbreaks following contamination of soap, particularly in critical patient groups, such as newborns and transplantation recipients. ${ }^{4,7}$

In this study, we aimed to evaluate the microbial contamination of "in use" soaps and the clonal relatedness of the soap-contaminating microorganisms in our hospital, a 450bed university hospital in Turkey. This research was performed with the approval of the university's Training and Research Hospital Ethics Committee. We performed cultures of samples from 383 soaps that were in use in our hospital during a 1-week period. For each soap included in the study, we used a form to indicate the type of soap (liquid or bar), the unit and the room in which the soap was used, the date and hour at which samples for culture were taken, the time the container was last replenished with fresh soap, and the population using the soap. Bacterial and fungal cultures were performed. The clonal relatedness of the isolates obtained from soaps was assessed using pulsed-field gel electrophoresis (PFGE) of the genomic DNA, as described elsewhere. ${ }^{9}$ Statistical analyses were performed with SPSS, version 11.0 (SPSS), using the $\chi^{2}$ test.

Of the 383 soaps, 378 were liquid and only 5 were bars. Also, 361 of the samples were from soap in general use (which does not contain a germicide), and the remaining 22 samples were from the private soaps of patients. Bacterial growth was found in 44 (11.4\%) of the soaps (all liquid); 1 bacterial isolate came from an antibacterial-containing private liquid soap of a patient. A single microorganism was isolated from 43 of the 44 positive samples, whereas one yielded 2 different bacteria. No growth was observed on fungal cultures. No contamination was found in the original container or the plastic cans used to distribute the soap, demonstrating extrinsic contamination of the soaps during use. The organisms isolated from the soaps were $P$. aeruginosa (16 isolates), Enterobacter aerogenes (9), E. coli (8), K. pneumoniae (6), Enterobacter cloacae (3), S. marcescens (2), and Klebsiella oxytoca (1).

By unit, the proportion of samples that yielded microorganisms on culture was as follows: 6 of 16 from the ophthalmology ward, 6 of 15 from private clinics, 4 of 9 from the dermatology ward, 3 of 16 from the pediatrics ward, 3 of 20 from the physical medicine and rehabilitation ward, 3 of 39 from the obstetrics and gynecology ward, 2 of 9 from the emergency department, 2 of 5 from the gastroenterology ward, 2 of 10 from the dialysis unit, 2 of 7 from the cardio- 